\title{
Frontières
}

\section{Le deuil blanc du parent âgé souffrant de démence}

\section{Michel Pozo}

Volume 16, numéro 2, printemps 2004

Deuil, blessure vive

URI : https://id.erudit.org/iderudit/1074111ar

DOI : https://doi.org/10.7202/1074111ar

Aller au sommaire du numéro

Éditeur(s)

Université du Québec à Montréal

ISSN

1180-3479 (imprimé)

1916-0976 (numérique)

Découvrir la revue

Citer cet article

Pozo, M. (2004). Le deuil blanc du parent âgé souffrant de démence. Frontières, 16(2), 22-27. https://doi.org/10.7202/1074111ar

\section{Résumé de l'article}

Les pathologies démentielles font essentiellement deux victimes : la personne qui est atteinte, mais aussi un membre de sa famille, conjoint ou enfant, qui, en tant qu'aidant principal ou de tuteur privilégié, partage au quotidien le drame de cette maladie et en porte en priorité la lourde charge, le plus souvent avec dévouement et courage. À un stade avancé, le dément renvoie une image de mort psychique, qui précéderait en quelque sorte la mort physique. Le caractère dégénératif et irréversible de la démence va entraîner un véritable bouleversement psychique chez l'enfant dont le parent est atteint par cette pathologie, notamment par un fonctionnement relationnel qui s'inverse et une réactivation des positions oedipiennes, aboutissant à une sorte de deuil avancé, qualifié de « deuil blanc " chez l'enfant, le poussant à se détacher affectivement du parent pour réduire son angoisse et sa souffrance.
Ce document est protégé par la loi sur le droit d'auteur. L'utilisation des services d'Érudit (y compris la reproduction) est assujettie à sa politique d'utilisation que vous pouvez consulter en ligne.

https://apropos.erudit.org/fr/usagers/politique-dutilisation/ 


\section{Résumé}

Les pathologies démentielles font essentiellement deux victimes : la personne qui est atteinte, mais aussi un membre de sa famille, conjoint ou enfant, qui, en tant qu'aidant principal ou de tuteur privilégié, partage au quotidien le drame de cette maladie et en porte en priorité la lourde charge, le plus souvent avec dévouement et courage. À un stade avancé, le dément renvoie une image de mort psychique, qui précéderait en quelque sorte la mort physique. Le caractère dégénératif et irréversible de la démence va entraîner un véritable bouleversement psychique chez l'enfant dont le parent est atteint par cette pathologie, notamment par un fonctionnement relationnel qui s'inverse et une réactivation des positions œdipiennes, aboutissant à une sorte de deuil avancé, qualifié de " deuil blanc » chez l'enfant, le poussant à se détacher affectivement du parent pour réduire son angoisse et sa souffrance.

Mots clés : démence - famille - parent enfant-deuil.

\begin{abstract}
Dementia pathologies essentially create two victims: the person afflicted with the illness and a member of the family, a spouse or child, who, in the capacity of principle caretaker or tutor, shares the daily drama of the illness and essentially assumes the brunt of the responsibilities with dedication and courage. In its advanced stages, dementia serves as an image of psychic death, which appears as a harbinger of the physical death to come. The degenerative and irreversible character of dementia can bring about a psychic upheaval for the child whose parent is touched by such an illness, notably through a reversal of the relational functioning and a reactivation of the oedipal positions, ending in an advanced type of mourning, called "deuil blanc" in children, forcing the child into an affective distancing to reduce both suffering and anguish.
\end{abstract}

Key words: dementia - family - parentchild-mourning.

\section{LE DEUIL BLANC DU PARENT ÂGÉ SOUFFRANT DE DÉMENCE}

\section{“ L'ANCÊTRE INSUFFISAMMENT BON ${ }^{1}$ »}

JOUBERT, 1995.

\section{Michel Pozo, \\ psychologue et gérontologue, Lyon, France.}

Si la démence apparaît comme le problème central de la clinique du grand âge, ce n'est pas par hasard. Les épidémiologistes nous livrent un élément de réponse en nous enseignant que trois facteurs concourent à l'augmentation de sa prévalence. Tout d'abord, l'avancée en âge puisque son incidence annuelle croît de façon exponentielle avec l'âge. Ensuite, l'espérance de vie des patients, car elle serait peu différente des sujets sains du même âge. Enfin, l'attention actuelle portée à la maladie d'Alzheimer aussi bien dans le public qu'au niveau médical. Mais, par-delà ces considérations scientifiques et socioéconomiques, se profile un argument purement psychologique lié à l'intolérable et à l'insoutenable de la démence.

La démence est un drame humain : drame pour les soignants qui en ont la charge, drame pour la médecine qui ne dispose d'aucun remède pour la combattre, drame pour la société qui n'est pas préparée à un tel problème socioéconomique, mais principalement drame enfin pour le patient et sa famille dont les liens sont chaque jour à reconstruire: "La démence est une pathologie du désespoir articulée avec une dépression familiale », souligne J. Maisondieu (1989).

La pathologie démentielle se définit en effet par une altération progressive et irréversible de l'ensemble des fonctions supérieures : mémoire, orientation dans le temps et l'espace, attention et jugement, langage, praxies (capacité d'accomplir des gestes et des actes) et gnosies (reconnaissance des objets, identification des parties du corps et du visage). Cette atteinte s'étend à l'ensemble de la vie psychique, accompagnée par des troubles du comportement, de l'affectivité et de la pensée, et transforme finalement le malade en un être humain de plus en plus dépendant, qui verra menacer jusqu'à sa capacité de sourire (Gil, 1989).

\section{FAMILLES ET PARENTS ÂGÉS}

La famille correspond à l'ensemble des individus liés par un lien de parenté et dont l'ensemble basal est constitué par le père, la mère et les enfants. La notion de famille, du moins dans notre culture, est généralement moins restrictive et s'étend de façon permanente aux grands-parents. En France, par exemple, cet aspect traditionnel se retrouve dans la législation actuelle qui stipule le devoir alimentaire des enfants mais aussi des petits-enfants vis-à-vis des parents.

La famille est le creuset du tissu relationnel de l'individu. À l'instar du tissu cutané, du tissu cérébral ou de tout autre tissu organique, il semble opportun de parler de tissu relationnel, indispensable à l'homme pour une vie raisonnable. C'est dans le cadre familial que ce tissu apparaît, se développe et se façonne. Toute lésion de ce tissu peut aboutir à de véritables hémorragies affectives. Les médecins connaissent bien les risques encourus à institutionnaliser un sujet notamment trop jeune ou trop âgé.

La famille est évolutive et elle est un organisme vivant. Les rapports entre 
ses membres qui définissent la place et le rôle de chacun sont déterminés par des paramètres qui diffèrent d'une société à l'autre. Rien n'est acquis. L'autorité, la référence, le pouvoir peuvent être déterminés par la croyance, l'âge, le sexe, le savoir, la force physique... Les personnes âgées ont ainsi eu une place variable dans la famille. Prépondérantes, honorées, adulées, elles peuvent également être rejetées, méprisées.

Le passage d'une société de type rural à une société à développement industriel, d'une part, et l'accroissement de la population âgée de plus de 65 ans, d'autre part, ont été déterminants pour l'évolution de la structure familiale et de son rapport à la personne âgée. En effet, pour la première fois dans l'histoire, deux générations adultes, parents et enfants, coexistent longuement. On assiste à ce que les démographes appellent «l'emboîtement des générations ».

Les relations de la personne âgée avec sa famille constituent un vaste champ d'investigation et, pour cet article, nous avons choisi de privilégier l'analyse des relations entre les parents âgés et leurs enfants. Ces relations se déroulent normalement tant que la personne âgée conserve un rôle à l'intérieur de la famille, que ce soit au moyen d'échanges de services, d'une solidarité entre les générations. Le rôle le plus fréquemment décrit est celui de grandsparents où les parents âgés retrouvent avec leurs petits-enfants une relation gratifiante et empreinte de complicité (Mishara et Riedel, 1994).

En fait, les difficultés commencent lorsque le parent âgé se dégrade, qu'il perd tout contrôle à l'intérieur de la famille, qu'il nécessite une attention constante de la part des enfants. Progressivement, on assiste à une prise en charge des parents par les enfants, prise en charge aussi bien matérielle qu'affective qui est supportée un temps par la famille jusqu'à un point de rupture (affection somatique, chute au domicile...) qui précipite la prise de conscience par les enfants que le parent âgé ne peut plus se suffire à lui-même.

\section{LE « FARDEAU » \\ DE L'AIDANT NATUREL}

D'après une estimation de la Fondation nationale de gérontologie, à 65 ans, 1 personne sur 20 présente des signes d'atteinte démentielle et à 80 ans 1 sur 5 (Charazac, 1998). La majorité des personnes souffrant de démence vivent à domicile, dans une proportion de $88 \%$ et, dans 50 à $60 \%$ des cas, leur prise en charge parfois très lourde est assumée par la famille, sans aucune aide des professionnels (Featenu, 1991).

Les descendants font partie des aidants naturels, ceux qui « légitimement» devraient subvenir aux soins de leur parent ou leur conjoint. Légitimement, selon une homéostasie de circulation des aides au sein du groupe familial. Les solidarités familiales se maintiennent et prennent source dans la dette symbolique que les enfants ont (ou pensent avoir) vis-à-vis de leurs parents. Cette notion de dette, induite par le don (initial) de la vie par ses géniteurs, le don affectif et toutes les autres formes de soutien, impliquerait des rapports intergénérationnels, principalement entre l'âgé et l'enfant adulte, fondés sur la réciprocité, l'équité et le devoir de rendre à son tour pour s'affranchir de la dette contractée envers ses parents. "Quand j'étais petit, tu m'as soigné quand j'étais malade, c'est à mon tour maintenant » dans une sorte de dette remboursée. «Je me suis occupé de mes parents, mes enfants s'occuperont de moi » dans une circulation de la dette à la génération suivante.

Les aidants naturels sont inscrits dans une transgénérationnalité du sens de la famille. En revanche, les soignants sont des aidants non naturels, dans le sens où ils ne sont pas naturellement désignés pour accomplir cette tâche, ceci pour bien marquer la différence avec la famille et faire ressortir le côté délicat de la position de

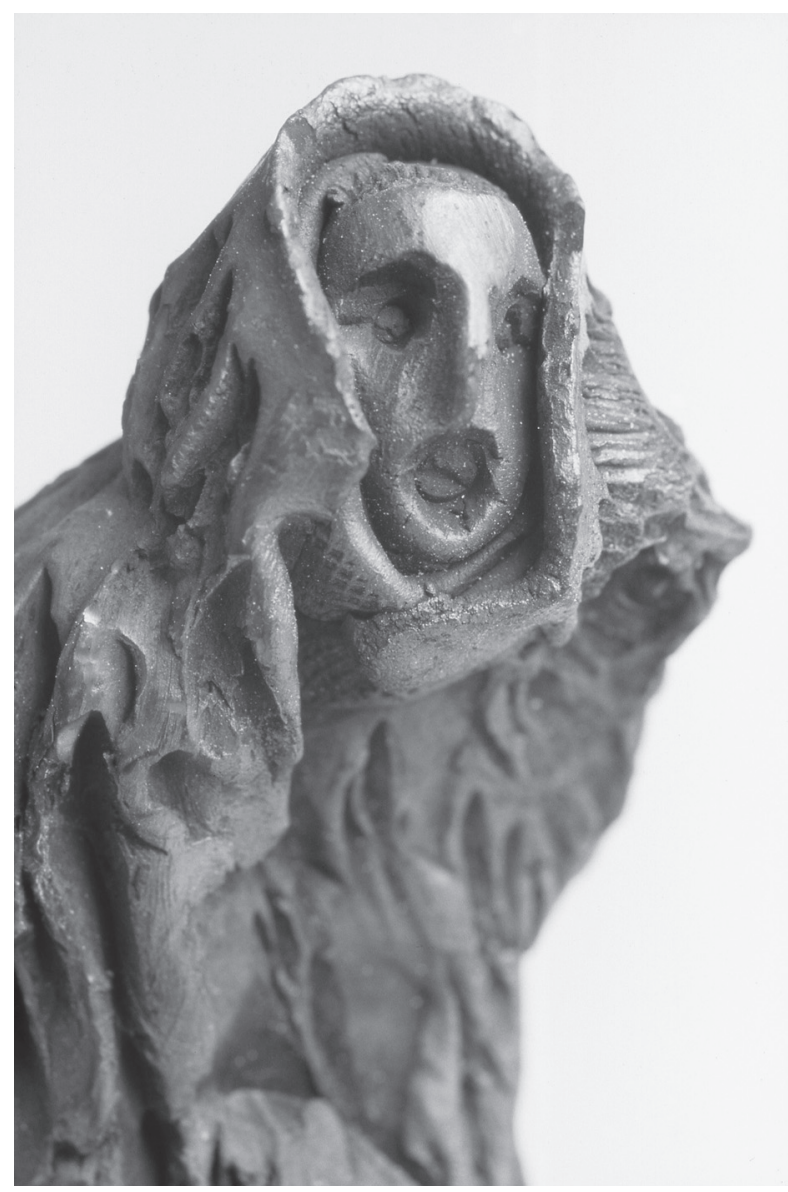

soignant. Ils ne font pas partie de cette famille naturelle : ils ne connaissent ni les dettes, ni les dons circulant dans cette cellule familiale.

Le transgénérationnel n'est pas leur affaire: ils peuvent, à la limite, s'inscrire dans une intergénérationnalité de type social et non familial. C'est ce que nous appelons la solidarité nationale. Dans l'histoire de la prise en charge des personnes âgées, celle-ci est longtemps restée une affaire privée qui concernait les membres de la famille et donc se faisait «naturellement » pour la majorité des cas. L'évolution de la société et les progrès de la médecine posent différemment le problème des personnes âgées : les aidants naturels ne peuvent pas toujours répondre à cette demande de prise en charge des aînés. Ce phénomène s'accentue quand la question de la dépendance vient compliquer quelque chose de naturel.

Selon une enquête de la Caisse nationale d'assurance vieillesse réalisée en 1990, la dépendance concerne $29 \%$ des 75 ans et plus. Parmi eux, $60 \%$ reçoivent une aide informelle de l'entourage. Ce sont surtout les femmes qui sont désignées ou s'autodésignent comme aidants naturels (une fille dans $63 \%$ des cas, et une belle-fille dans $9 \%$ ). Seuls $29 \%$ des fils s'occupent de leurs parents. Il s'agit en général de fils célibataires. L'appel à l'aide extérieure est plus important d'ailleurs quand il s'agit de fils-aidants : $35 \%$ contre $22 \%$ des filles-aidantes. Cette prévalence va avoir des conséquences psychologiques dans les relations avec les professionnels appelés. En effet, la majorité du personnel soignant est en fait constitué de femmes.

Les Américains évaluent ainsi à 6 millions le nombre de personnes concernées par le family burden du maintien à domicile du parent. Cette expression qui signifie littéralement fardeau, souci, inquiétude, peut aussi se traduire par charge familiale, terme plus neutre puisqu'il désigne aussi les soins.

Ce fardeau a été mesuré : en ajoutant le temps des activités directement liées à l'état du malade (toilette, ๘ habillement, repas, soins d'hygiène, que ce soit en effectuant ces activités ou en surveillant leur bon déroulement) et toutes les tâches matérielles de la vie de la maison, on arrive aux 50 ou 60 heures par semaine dans ce rôle d'aidant qui est presque toujours assumé par un membre de la famille (Petit, 2002).

Mishara (Mishara et Riedel, 1994) distingue la charge objective, à savoir les conditions matérielles de la 
cohabitation, et la charge subjective, c'est-àdire le retentissement physique et psychologique attribué à cette vie quotidienne.

Les différents travaux décrivent le même ensemble de réactions ou de troubles chez le caregiver, c'est-à-dire le membre de la famille qui vit avec le parent dément ou passe le plus de temps avec lui : stress, surmenage et altération de la santé physique ; déni de la maladie du parent ou des problèmes qu'elle pose.

Donaldson et Burns (1999) soulignent que lorsqu'un aidant sent que la tâche dépasse sa capacité à y faire face, il se sent stressé tandis que la même tâche effectuée par un aidant se sentant armé pour le faire ne le sera pas. Cette même étude révèle que des variables peuvent interférer sur le stress, telles que son âge, son sexe, son mode de parenté (conjoint, enfant), son organisation de vie, mais aussi des variables liées à sa personnalité, telles que ses tendances anxieuses ou dépressives préexistantes, la qualité de la relation au parent, l'aide qu'il reçoit de ses proches et de la société. Mais la variable la plus déterminante tient à sa façon d'attribuer les symptômes à la maladie du patient ou au patient lui-même. Dans ce dernier cas, l'aidant serait amené à penser qu'ils sont volontaires, délibérés et sous contrôle ( Il le fait exprès »). Ce qui peut l'inciter à croire que certaines manifestations sont contrôlables par le patient, c'est leur caractère intermittent, et notamment l'agressivité qui s'exerce tout particulièrement envers l'aidant. Il est donc important que l'aidant prenne conscience, dès le début, que nombre de manifestations qu'il supporte difficilement sont en lien avec le processus pathologique, c'est-à-dire avec une cause externe de contrôle, plutôt qu'attribuable au malade lui-même.

Les travaux d'É. Kübler-Ross (1975) sur la survenue de la maladie grave chez des patients incurables ont été repris par Teusink et Mahler (1984). Ils ont pu distinguer cinq étapes dans les réactions des familles :

1. Le déni des troubles: la famille remarque les troubles de mémoire, mais les banalise en les mettant sur le compte de l'âge.

2. Le surinvestissement: les membres de la famille s'efforcent de compenser l'atteinte du parent devenue importante, en sacrifiant leur vie personnelle, sans pour autant s'autoriser à demander de l'aide à des intervenants extérieurs.

3. La colère : ce sentiment, transféré fréquemment sur les professionnels qui essaient d'apporter leur aide à la famille, est à la fois une réaction à l'alourdissement des symptômes du parent, mais également un sentiment d'avoir été abandonné par le parent.

4. La culpabilité : qui succède à la colère ou au souhait de la mort du parent.

5. L'acceptation: lorsque l'entourage a compris le caractère irréversible de la maladie qui touche le parent et accepté que l'être aimé ne soit plus la personne qu'il a connue jadis.

K. Ritchie et al. (1992) ont également souligné, sur la base de diverses études de la littérature anglo-saxonne, l'augmentation d'incidence de la pathologie mentale et physique chez les aidants de personnes démentes, ainsi que leur surconsommation en psychotropes, même lorsqu'ils n'ont plus directement à charge le malade après son entrée en établissement gériatrique. Les auteurs ont réalisé une enquête auprès des aidants naturels des personnes démentes hébergées dans un service hospitalier : $40 \%$ d'entre eux se disent en mauvaise santé et $53 \%$ se disent déprimés. Les résultats de cette étude sont corrélés par ceux constatés par Haley (1997) : 30 à 50\% des aidants familiaux présentent des signes de dépression, et une surconsommation médicale et pharmaceutique. Cela, malgré la rareté d'histoire antérieure de dépression chez la plupart.

\section{LE FONCTIONNEMENT RELATIONNEL INVERSÉ}

Être l'enfant d'un parent souffrant de démence est ainsi une tâche ardue à bien des égards. L'enfant doit tout d'abord affronter les difficultés physiques et surtout psychiques de la relation avec son parent. Mais ici la relation est d'autant plus contraignante qu'elle est de longue durée et d'autant plus complexe qu'elle est nécessairement parasitée par un lien affectif intense et ancien. Les sollicitations du parent âgé sont pesantes ou vécues comme telles. Il faut dire que la demande est souvent ambiguë. Le vieillard veut souvent garder le commandement (position parentale) tout en se faisant choyer (position régressive donc infantile). La revendication affective est présente en filigrane avec l'attente d'être payé en retour de ses efforts passés vis-à-vis de ses enfants, et surtout une demande inassouvissable de réassurance narcissique, lorsqu'il n'a pas pu, sa vie durant, acquérir le sentiment d'une identité suffisamment solide.

Un autre aspect de cette relation est une troublante inversion des rôles. Le vieillissement des parents facilite l'instauration d'une prise en charge des parents par les enfants. Si l'on reprend l'histoire de la relation parents-enfants, la première relation est fondée sur la dépendance totale de l'enfant par rapport à ses parents, aussi bien pour sa survie matérielle que pour son équilibre affectif. Lorsque l'enfant grandit, il prend progressivement son autonomie, partielle tout d'abord, puis totale au moment où il quitte sa famille pour créer à son tour une famille. Au moment du vieillissement de ses parents, on assiste à une relation inversée dans la mesure où ce sont les parents qui deviennent dépendants. En effet, il suffit d'une situation de rupture (affection somatique ou pathologie démentielle) pour que les enfants prennent totalement en charge les parents. La prise de conscience par les enfants que le parent âgé ne peut plus se suffire à lui-même induit de leur part une relation d'autorité et de pouvoir qui répète les premières relations en sens inverse. À cela, il convient d'ajouter la difficulté angoissante et culpabilisante de décider à sa place quand il a perdu ou abandonné son autonomie.

L'enfant se voit désigné ou se situe de lui-même pour la première fois comme répondant, responsable, "tuteur privilégié » selon les formules consacrées (Andrieu, Bocket, Nourhasi et al., 1999).

Tendresse, amour, juste retour des choses, règlement de compte, devoir, dette, contrainte coercitive ou compulsive..., quelque part la situation du parent vieillissant touche, mobilise et pousse à devenir «bâton de vieillesse ». Un agir, des attitudes, bref, un comportement dit «naturel» ou « normal» ou « allant de soi » et qui renvoie à une inscription familiale s'enracinant profondément dans l'histoire personnelle de chacun.

Cette inversion acceptée ou refusée, culpabilisante ou paniquante, déstabilisante de toutes les façons et renforçant ce vieux dicton: «les parents âgés sont les enfants que l'on a sur le tard», c'est le «je suis devenue la mère de ma mère ».

Toute une famille est en quête de repères. Derrière la tendresse et l'affection, et surtout si l'on s'entend bien en famille, sont remis en question le pouvoir, l'autonomie, et la place de chacun dans la hiérarchie familiale. Sur des tonalités anciennes se joue avec bonheur ou souffrance la partition d'accompagnement de la toute dernière étape de vie du parent âgé. Certains s'y bousculent, comme le montre la rivalité de certains enfants autour de leur mère, d'autres la fuient, c'est selon.

Dans le soutien à l'âgé, tous les liens de parenté peuvent se mettre en scène: conjoint / conjointe, parent / enfant, grandparent / petit-enfant, beau-parent / bellefille ou gendre, frère / sœur, tante ou oncle / neveu ou nièce, cousin / cousine. Prédomine néanmoins, comme nous le signalions plus haut, le lien parent-enfant et plus précisément mère-fille.

À ce propos, comme le souligne I. Simeone (1989), il est intéressant de noter que si gériatrie est un mot qui se prononce au 
féminin, comme démence, il est vrai que c'est une «langue » parlée au quotidien par des femmes: les patientes, les femmes de leurs familles, les soignantes. Face à cette réalité, on peut se demander si l'homme ne prend pas d'ailleurs la fuite. Ou s'il ne se propose pas, est-ce parce qu'il y a une femme qui, tout naturellement, se met en avant, et offre des soins «maternels »? Car il est vrai que la personne souffrant de démence réclame essentiellement des soins maternants.

Cette mise en acte du fantasme de renversement de l'ordre des générations peut être renforcée par la personne démente, notamment lorsqu'il y a confusion entre sa propre mère et sa fille: Mme V. en me montrant sa fille, me dit: "Je vous présente ma mère ", ou encore Mme D. accueillant sa fille: "Maman, te voilà enfin! » Pour C. Montani (1994), les descendants, happés dans le filtre d'une reconstruction fantasmatique de la filiation par la personne démente, viennent incarner, c'est-à-dire prendre la place des ascendants disparus.

\section{LA RÉACTIVATION DE LA SITUATION CEDIPIENNE}

Pour C. Joubert (1996), le fantasme de mort collective mis en évidence par A. Ruffiot (1985) au sein des familles à fonctionnement psychotique est très présent également dans les familles ayant un de leurs parents atteint de démence. Le fantasme de mort collective est en lien avec le déni de la mort individuelle.

Nous croyons en effet, par notre pratique clinique, que la personne âgée dégradée et dépendante est «porteuse de mort»: elle incarne la mort et devant le parent très âgé, l'enfant qui vieillit se trouve confronté avec sa propre mort, ce qui l'angoisse. Combien de fois recevons-nous des familles qui se disent épuisées par la maladie du parent, déprimées, et qui décident un placement en maison de retraite car « elles ne peuvent plus faire face, elles vont y laisser leur peau ... » Ainsi s'exprimait Mme O., demandant une entrée en institution pour sa mère de 85 ans : "Tous ces trajets que je fais, aller la voir tous les jours, je n'en peux plus, je vais y laisser ma santé... »Comme si le parent âgé «vidait» l'enfant de toute son énergie, l'affaiblissait, risquait de le rendre malade, ou de le faire mourir. L'angoisse qui envahit alors l'enfant est de nature persécutive. Il se sent attaqué, étouffé, parfois dévoré par son parent qui devient mortifère.

Le symptôme présenté par la famille véhicule la mort non tant pour le parent âgé que pour l'entourage. Lorsqu'il demande un placement, c'est pour éloigner la mort, la maintenir à distance. Et, dans ce placement, il réalise une petite mort à l'égard de son parent, il le fait un petit peu mourir et il accomplit ce désir de mort en même temps qu'il le camoufle car il se sent très coupable. Ce désir de mort à l'égard des parents répète une situation que l'enfant a déjà connue. En effet, l'enfant, que tout adulte a en lui, a déjà eu ce désir de mort au moment de la situation œdipienne. Le complexe d'Edipe est, au plan inconscient, l'ensemble des désirs amoureux et hostiles que l'enfant éprouve à l'égard de ses parents avec un désir de mort pour le parent du même sexe et un désir sexuel, mais coupable, pour le parent du sexe opposé. Sans expliciter à nouveau le conflit œdipien, on peut penser que lorsque le fils grandit et que le père se dégrade, cette situation se renouvelle, mais cette fois c'est le fils qui a le pouvoir.

Le père vieilli se trouve en position inférieure et son placement par le fils peut être analysé comme une revanche du fils, un meurtre du père, une sorte de liquidation tardive de la situation œdipienne. Cependant, ce désir de mort à l'égard du parent du même sexe s'accompagne d'une grande culpabilité. L'agressivité et la culpabilité sont toujours très proches comme en témoigne l'agressivité des familles dans les institutions ; cette agressivité est à l'exacte mesure de leur culpabilité. Pour M. Myslinski (Myslinski et Simeone, 1986), qui a beaucoup écrit sur les relations entre fille et mère démente,

la mère devient une mauvaise mère, associée à la vieillesse, qui devient donc une mauvaise vieillesse. De rassurante, l'image maternelle devient persécutoire, de même que l'image de la vieillesse. Le clivage, la séparation s'opère entre l'objet extérieur - l'objet réel, la personne de la mère - et l'objet maternel interne - la Mère incorporée depuis l'enfance, ayant fonction depuis toujours de réassurance.

C'est la situation de Mme P. qui nous dit : « Ma mère n'est plus ma mère. Elle n'est plus comme avant, elle est devenue folle. Elle me rend folle, elle qui était si douce et si gentille. Elle ne me reconnaît même plus. »

Il résulte chez la fille le sentiment d'avoir détruit, perdu le bon objet, et par conséquent, de le restaurer, de reconstituer la bonne mère. D'où cette disponibilité, cette patience sans répit, accordées à la mère tout au long des jours et des années, au détriment des enfants, du conjoint et de soi-même.

À l'opposé, avec le parent du sexe opposé (le fils plaçant sa mère, la fille plaçant son père), l'enfant reste très ambivalent, cherche des réassurances multiples pour cautionner sa démarche et éprouve une profonde culpabilité d'abandonner celui ou celle qui fut son premier objet d'amour.

\section{LA SITUATION D'INTER-CRISE}

Nous pensons que les difficultés et les conflits de la famille avec le parent âgé peuvent également s'analyser comme une rencontre de plusieurs crises:

- la crise du grand âge chez le parent âgé ;

- la crise de la vieillesse : le déclin du parent âgé équivaut à une prise de conscience brutale de son propre vieillissement, et pour l'être abordant la vieillesse, c'est une confrontation dramatique avec un avenir fermé sans la protection magique contre la mort qu'assuraient jusqu'alors les parents ;

- la crise du milieu de la vie où la personne occupe sur l'axe temporel une place intermédiaire entre le parent âgé et dépendant, et l'enfant en phase d'adolescence ou de pré-adulte remettant en cause l'autorité parentale et la dépendance qui s'y rattache. En somme, on se retrouve devant quelqu'un contraint d'abandonner une relation d'autorité avec ses enfants pour la déplacer à l'égard de ses parents. C'est donc à un double deuil que la personne est confrontée: le deuil de ses parents ou tout au moins d'une image positive et le deuil de ses enfants, c'est-à-dire de la relation autorité-pouvoir.

Les difficultés des enfants sont ainsi d'autant plus prégnantes qu'elles surviennent à une période de leur vie délicate : c'est « la génération sandwich » dont parle L. Ploton (1990). Faire partie de cette génération, c'est être dans la tranche d'âge 50-70 ans. La jeunesse n'est plus et la vieillesse commence à se faire sentir. C'est un entredeux par rapport à des étapes temporelles définies : l'âge adulte et la sénescence. Ce temps que M. Myslinski appelle le temps de la «maturescence» présente des particularités. Sur le plan corporel, les premiers signes du vieillissement sont là. Rides, cheveux blancs, presbytie et surtout ménopause pour les femmes qui sont les interlocutrices privilégiées. Pour M. Trouilloud (1997), ces filles investissent d'autant plus un rôle de mère vis-à-vis de leur parent dépendant que leur difficulté à se penser ménopausée est grande et que l'autonomisation des enfants qui s'inscrit dans la réalité fait le lit d'un possible «syndrome du nid vide». Ces modifications corporelles s'accompagnent d'un vécu ou d'une crainte de perte de pouvoir de séduction, à mettre en lien avec la peur de ne plus être aimé, d'être laissé pour compte. Socialement, c'est le passage à la retraite, changement de statut 
important : l'actif productif doit apprendre à devenir passif bénéficiaire et se présenter en fonction de ce qu'il est, et non plus en fonction de sa profession.

Ces remaniements illustrent le temps de la maturescence, dont l'enjeu est le maintien de l'identité devant les changements. Selon J. Bergeret (1982), « il s'agit, sans renoncer à soi-même, à ses anciennes identifications satisfaisantes, de devenir un autre soi-même, en accord avec les nouvelles données de la vie, et d'intégrer une configuration de soi, autorisant l'estime de soi, et l'amour d'autrui ».

C'est dans ce contexte de crise d'identité que « la génération sandwich » est confrontée à la vieillesse avancée de ses parents. La grande dépendance d'un parent, en particulier dans le développement d'un syndrome démentiel, prive alors les enfants de repères possibles qu'aurait pu proposer un parent ayant traversé de manière estimable et paisible la dernière étape de sa vie : «le vieillard est devenu une représentation de la mort à venir au lieu d'être le survivant qui grâce à sa sagesse a échappé aux dangers de la vie », constate J. Maisondieu (1989).

\section{SOUFFRANCE DE L'AIDANT ET DEUIL ANTICIPÉ}

Enfant de son parent, on est enfant et on le reste toute sa vie durant (Viciana, 1997). On continue de s'identifier à son parent. C'est pourquoi il est difficile et parfois pénible de devoir faire face aux transformations physiques et psychiques de son parent dont on souhaite garder une image que le temps nécessairement altère. Si toutefois le parent a réussi son travail de vieillir, l'identification pourra continuer de s'effectuer dans de bonnes conditions, le parent achevant finalement le travail d'éducateur de son enfant en lui enseignant de façon exemplaire la vieillesse (l'art de vieillir pourrait-on dire). Mais que le vieillissement ne soit pas réussi, l'image de déchéance de son parent peut être difficilement supportable pour l'enfant qui en vient à se poser la question, parfois non dite, parfois exprimée dans un moment de détresse : «Ne vaudrait-il pas mieux qu'il-elle soit mort-e, plutôt que d'en être réduit-e à cet état? »

Mais la position d'un enfant par rapport à la mort future de son parent est profondément ambivalente. D'un côté, le parent âgé est vis-à-vis de lui le dernier rempart symbolique contre la mort ( « après lui, c'est moi » peut-on parfois entendre dire) : au point que, souvent, l'intervenant extérieur ne sait plus qui des deux s'agrippe à l'autre. Mais, d'un autre côté, ne lui faut-il pas conserver une image acceptable de lui, donc renoncer par avance à son existence ?

Finalement, un véritable travail de deuil du parent s'impose à l'activité psychique de l'enfant : deuil anticipé, c'est-à-dire démarrant avant le décès, et évoqué comme un sentiment de «mort sans mort » (Maisondieu, 1989).

Ainsi, au fil des jours, la souffrance se double d'un travail de deuils successifs qualifié par certains de « deuil blanc » (PancraziBoyer et al.,1996), mis en œuvre dans l'accompagnement d'un parent qui se démentifie :

- deuil de l'espérance de guérison, même si des progrès thérapeutiques existent;

- deuil de la relation perdue: «il ne me reconnaît plus »;

- deuil de ce parent idéal : «lui qui était si gentil, si fort, si intelligent»;

- deuil de l'estime de soi devant l'agressivité manifestée lors des comportements perturbateurs quotidiens du dément. Ne supportant plus la situation, certains se posent la question du sens d'une telle vie et souhaitent sa fin... tout en se reprochant un tel souhait.

La question obsédante d'une éventuelle hérédité de la maladie vient de plus obscurcir davantage sentiments et conduites, avec le refus d'un avenir identique au sien, alors qu'il a potentiellement transmis le mal à sa descendance. Souhaits de mort et peur qu'il meure se révèlent donc étroitement mêlés, à un degré de plus, la question du poids de sa responsabilité à assumer s'il lui arrivait réellement «quelque chose» (Ploton, 1996).

Ainsi, le parent âgé souffrant de démence, n'est pas réellement mort, mais perdu ou en train de se perdre. Ce parent est devenu autre. Quelque chose a changé le parent, et le proche ne s'y reconnaît plus. Telle mère active, qui avait soigné son mari, avait fait face aux séquestres de ses biens pendant la guerre, qui récemment encore voyageait à travers le monde... maintenant ne se nourrit plus, met une casserole sur le feu et l'oublie, ne veut plus faire ses courses, ne se lave plus alors qu'elle prétend le contraire... P. Charazac (1998) définit le symptôme démentiel comme une réalité à la fois étrangère et inquiétante (au sens de l'allemand Unheimlich dont l'une des traductions est «ne faisant pas partie de la maison »), qui acquiert une valeur traumatique au moment où la famille n'est plus capable de l'inscrire dans son histoire, se trouvant prise dans l'actualité de la maladie, les traumatismes passés qu'elle réactive et la pensée de la mort à venir du parent.

Dans un premier temps, confronté à l'altérité du parent, l'enfant se rebelle contre la réalité et tente de conserver le parent âgé dans son intégrité, comme le montre la minoration, voire la négation des troubles dans certaines familles. Puis, dans un deuxième temps, l'enfant reconnaît la trans- formation du parent. Celle-ci est vécue dans une dimension négative, destructrice. Cette altérité du parent âgé souffrant de démence entraîne pour le proche une modification dans la relation d'amour. Nous pourrions dire dans certains cas que le parent est perdu en tant qu'objet d'amour, comme le souligne S. Freud (1985 [1917]) : « l'épreuve de la réalité a montré que l'objet aimé n'existe plus et édicte l'exigence de retirer toute la libido des liens qui la retiennent à l'objet ».

La notion de perte d'objet d'amour nous fait ainsi entrer dans les problématiques du deuil et de la dépression. En effet, S. Freud (1985 [1917]) et M. Klein (1989 [1947]) montrent les liens entre la dépression et la relation d'objet. La dépression étant suscitée par la perte de l'objet aimé : soit par une intervention interne, par exemple, les pulsions destructrices du sujet envers l'objet d'amour; soit par une intervention externe comme un décès, et nous pourrions ajouter, l'altérité de l'objet d'amour due à une affection acquise, telle que la démence. Parfois on assiste à un relâchement des relations entre parents âgés et enfant comme si cette mise à distance permettait à l'enfant de ne pas souffrir d'une identification à un support qui s'effondrerait. En effet, l'enfant a toujours le souvenir d'une relation positive embellie, car le temps de l'enfance apparaît souvent idyllique. Or, l'image des parents détériorés est narcissiquement insupportable dans le sens où elle demande à l'enfant de faire le deuil de cette image valorisante.

Quant au parent âgé, il maintient le plus souvent l'illusion d'une relation positive avec ses enfants comme si le deuil d'une relation gratifiante était impossible. Même lorsque les enfants sont à l'origine du placement, les parents âgés en font souvent la dénégation en faisant assumer la responsabilité à un tiers et aménagent la réalité selon leur désir: "ce ne sont pas mes enfants qui m'ont mise ici, ils vont me prendre chez eux ».

L'essentiel est que ce processus de deuil anticipé ne trouve pas son terme avant la mort du parent, sinon le parent sera réellement abandonné, et l'enfant risquera de se le reprocher toute sa vie. En définitive, l'enfant est soumis à une double contrainte : se détacher de son parent tout en continuant de s'attacher à lui. Contradiction bien difficile à maîtriser et source d'un conflit psychique insupportable. Une lutte permanente entre un amour qui ne cède pas pour l'aimé perdu et une force qui nous détache de lui. « La douleur du deuil n'est pas douleur de séparation, mais douleur de liaison : penser ce qui fait mal, ce n'est pas de se séparer, mais de s'attacher plus fort que jamais à l'objet perdu », conclut J.D. Nasio (1997).

$$
* * *
$$


L'irruption de la démence dans la famille peut faire l'effet d'un cataclysme, qui va bouleverser l'équilibre établi, tous ces compromis tacites entre les uns et les autres, qui font que chacun a une certaine place, un certain rôle. Si l'on souhaite éviter un certain nombre de crises familiales nocives pour le parent âgé (régression, maltraitance, placement...) mais aussi pour le reste de la famille (culpabilité, épuisement, dépression, arrêt de travail...), il semble nécessaire de proposer un certain nombre de solutions face à ces difficultés.

Le climat relationnel de tous les intervenants autour de la famille est tout à fait fondamental. L'aidant principal doit être d'emblée repéré, afin d'être pris en charge en même temps que le malade lui-même. Le suivi devra porter conjointement sur les deux personnes. Dans une relation de confiance, il faut accompagner ce travail de deuil blanc, pour tenter de lui faire accepter l'idée que son parent n'est plus tout à fait le même avec la maladie, qu'il lui faudra s'adapter à lui.

Il est important qu'il puisse accepter des aides, et qu'il apprenne à se reposer sur des personnes de confiance. En France, le rôle de l'infirmière libérale auprès de la famille revêt une importance cruciale lorsqu'il lui permet de devenir une sorte de confidente de tous les malheurs. Il convient également de recenser les moyens matériels et humains qui peuvent aider à tisser autour de l'aidant une sorte de filet de sécurité, particulièrement indispensable lors des moments difficiles de l'évolution de la maladie. Des moments de récupération doivent être aménagés dès les premiers signes d'épuisement sous forme d'accueil de jour et de nuit, d'hospitalisation de jour, d'hébergement temporaire...

Les proches ont besoin d'informations, de conseils pratiques pour gérer les petits problèmes quotidiens (Khosravi, 1995), d'écoute bienveillante, et de partage avec d'autres familles: c'est le rôle des associations de famille, de type Association France Alzheimer, présente et active dans toutes les régions. Ces associations fonctionnent également en groupe de paroles, libérant de fortes charges émotionnelles. Certains établissements gériatriques organisent des réunions de famille, qui ont alors la même fonction.

Rencontrer la famille permet cette approche historique des personnes âgées qui, dans la démence, ne peuvent plus se raconter, mais il permet aussi et surtout de situer la personne âgée dans le champ du désir de l'autre et d'appréhender les interrelations entre cet ancêtre insuffisamment bon et sa famille.

Pour conclure, ces mots d'A. ComteSponville (1999) :
Toutes ces années qu'on a passées à s'opposer à son père ou à sa mère, à rivaliser avec eux, ce long combat qui n'en finissait pas, il sera donc sans vainqueur ni vaincu. Petits, nous étions trop faibles pour l'emporter. Jeunes, trop impatients, trop immatures, trop inachevés. Il nous aura fallu une vie pour devenir à peu près ce que nous voulions être, pour nous bâtir, nous fortifier - pour grandir. La victoire se profilait enfin à l'horizon. Trop tard. Celui qu'on voulait vaincre n'est plus en état de combattre, de résister, ni même d'être vaincu. Il n'y a plus que la mémoire, pour ceux qui l'ont gardée, et ce qu'on porte en soi d'amour, de gratitude ou de pardon.

\section{Bibliographie}

ANDRIEU, S., H. BOCQUET, F. NOURHASI et al. (1999). "La carrière de l'aidant informel », La revue de gériatrie, vol. $24, \mathrm{n}^{\circ} 2$, p. 133-143.

BERGERET, J. (1982). "La deuxième crise d'adolescence », dans J. GUILLAUMIN et H. REBOUL (dir.), Les dynamismes du vieillissement, Lyon, Chroniques Sociales, p. 71-78.

CHARAZAC, P. (1998). Psychothérapie du patient âgé et de sa famille, Paris, Dunod.

COMTE-SPONVILLE, A. (1999). "Face à face avec Alzheimer », Site : Psychologie.com.

DONALDSON, C. et A. BURNS (1999). «Family under stress : interventions for caregivers of senile dementia patients », Journal of Geriatric Psychiatry and Neurology, $\mathrm{n}^{\circ} 12$, p. 21-28.

FEATENU, D. (1991). «Déments et institutions », Confrontations Psychiatriques, $\mathrm{n}^{\circ} 33$, p. 393-408.

FREUD, S. (1985 [1917]). «Deuil et Mélancolie », dans Métapsychologie, Paris, Gallimard, 1985, p. 41-115.

GIL, R. (1989). « La détérioration intellectuelle et les syndromes démentiels », dans Neurologie pour le praticien, Paris, SIMEP, p. 139-148.

HALEY, W.E. (1997). «The family caregiver's role in Alzheimer's disease », Neurology, $\mathrm{n}^{\circ} 48$, p. 25-29.

JOUBERT, C. (1996). « Vers une gestion groupale de la démence sénile en institution. Prise en compte de la souffrance groupale, familiale, individuelle, dans le soin ", Psychothérapie des démences, Paris, John Libbey Eurotext, p. 171-178.

JOUBERT, C. (1995). «Famille et gériatrie: l'ancêtre insuffisamment bon", Psychologie Médicale, $\mathrm{n}^{\circ}$ 27, p. 223-226.

KHOSRAVI, M. (1995). La vie quotidienne du malade d'Alzheimer, Paris, Doin.

KLEIN, M. (1989 [1947]). "Le deuil et ses rapports avec les états maniaco-dépressifs », dans Essais de psychanalyse, Paris, Payot, p. 341-369.

KÜBLER-ROSS, E. (1975). Les derniers instants de la vie, Genève, Labor et Fidès.

MAISONDIEU, J. (1989a). Le crépuscule de la raison, Paris, Centurion.

MAISONDIEU, J. (1989b). « Lidentification impossible », Psychologie Médicale, vol. 21, $\mathrm{n}^{\circ} 11$, p. $1630-1632$.

MISHARA, B.L. et R. RIEDEL (1994). Le vieillissement (troisième édition révisée), Paris, Presses universitaires de France.

MONTANI, C. (1994). La maladie d'Alzheimer, Paris, L'Harmattan.

MYSLINSKI, M. et I. SIMEONE (1986). "Relation entre fille et mère démente au travers des groupes de soutien ", Psychologie Médicale, p. 1289-1290.

NASIO, J.D. (1997). Le livre de la douleur et de l'amour, Paris, Payot.

PANCRAZI-BOYER M.P., C. PETIT et R. ARNAUD-CASTIGLIONI (1996). «Groupe de soutien aux familles de patients atteints de la maladie d'Alzheimer. Réflexions préliminaires », Psychothérapie des démences, Paris, John Libbey Eurotext, p. 170-185.

PETIT, H. (2002). L'accompagnement dans la maladie d'Alzheimer, Paris, Phase 5.

PLOTON, L. (1990). La personne âgée, son accompagnement médical et psychologique et la question de la démence, Lyon, Chroniques Sociales.

PLOTON, L. (1996). Maladie d'Alzheimer, À l'écoute d'un langage, Lyon, Chroniques Sociales.

RITCHIE, K., C. NARGEOT et C. HERGUETTAT (1992). "Influence des éléments de perte d'autonomie dans la décision de placement », La revue de gériatrie, vol. 22, n ${ }^{\circ}$, p. 273-280.

RUFFIOT, A. (1985). "Originaire et imaginaire. Le souhait de mort collective en thérapie familiale psychanalytique », Gruppo, $\mathrm{n}^{\circ} 1$, p. 69-85.

SIMEONE, I. (1989). « Les affects de la famille: entre l'amour et la haine », Gérontologie et société, n ${ }^{\circ} 48$, p. 96-98.

TEUSINK, J.-P. et S. MAHLER (1984). «Helping families cope with Alzheimer's disease », Hospital and Community Psychiatry, vol. 35, $\mathrm{n}^{\circ} 2$, p. $152-156$.

TROUILLOUD, M. (1997). "Souffrance et épuisement psychiques des familles ", Revue de la fédération JALMALV, n 51, p. 9-13.

VICIANA, H. (1997). «Enfants à vie », Revue de la fédération JALMALV, n ${ }^{\circ}$ 51, p. 35-39.

\section{Notes}

1. Nous empruntons cette expression à Christiane Joubert (1995) : « Famille et gériatrie : l'ancêtre insuffisamment bon ». 